\title{
Protein Molecular Weight Computation from Sedimentation Velocity Data
}

\author{
J. GRIEVINK AND R. T. B. HOUTERMAN \\ Tremle Uninesitu of Technology, Enschede, The Netherlands
}

AND

K. I)H GROOT

Fiee University. Department of Material Science, Schools of Dentistry and Hedicine, de Roelelan 1115, Amsterdam, The Netherlands

Received April 19, 1973; accepted July 30, 1973

In whracentrifugation, the concentration gradient of mono-disperse sumples obtained by sedimentation velocity experiments is described by Gehatia's equation which holds several parameters including the sedimentation and diffusion constants. Onew thrse two constants are known, the molecular weight follows from the svedherg crutution. A least squares method has been develoned to derive the transport constants from the refractive index gradient curves. The method emploss a mathematical model based on Gehatia's theory. A main feature of the model is the application of two sets of intermediate parameters via which the transport coefficients are much casier calculated than along a direct way. Furthermore some difficult to observe quantities cancel out. The square residues are minimised numerically. The potential errors introduced by this numerical minimalisation are shown to be unimportant compared to the unavoidable experimental errors

\section{INTRODUTTION}

Molceular weight determination of proteins by means of ultracentrifugation usually proceds via cquilibrium sedimentation or the approach to equilibrium methor according to Archibald. Sedimentation velocity experiments are mostly analyzed using Fredberg's method, yielding scdimentation coefficionts $S$.

Gehatia (1), Gehatia and Hubner (2), (3) however, has provided methods to derive both $S$ and the diffusion constant $I$ ) and thus the molecular weight $H$, from a single sorlimentation volocity run on monodisperse samples. The advantages of obtaining molecular weights in

'To whom rorrespondence should he addressed.

Coprright (c) 1974 h Academir Press. Ine.

All rights of reproduction in any form reserved. 
Gehatia's way are obvious: the time needed for a velocity run is much smaller than the time neccssary for equilibrium or approach to equilibrium runs. Furthermore, the usual methods yield only $M$, not $s$ and $I$ ) separately.

The disadvantages, however, which have limited the practical use, are twofold. Firstly, Gehatia's method requires a few experimentally difficult to observe parameters such as the initial time $\left(t_{0}\right)$, the initial position of the phase boundary $\left(r_{0}\right)$. Sccondly, the arithmetical analysis necessary to obtain $S$ and $D$ is very cumbersome.

In this article a least squares method is described, employing a model that has been derived from Gehatia's theory (1). By means of two parameter transformations the determination of $r_{0}$ and $t_{0}$ can be bypassed. A scheme for automated data handling facilitates the execution of the tedious calculations considerably.

\section{THEORY}

The theory of sedimentation velocity experiments has been discussed extensively in the literature [Gehatia (1), Fujita (4)]. The results of Gehatia's analysis will be used as a starting point for the development of a model relating $S, D$ and $M$ to the measurable variables, i.e., refractive index gradient versus time and radial distance. This section describes the development of the model, while Sec. III deals with the application of the model to the data processing.

The sedimentation velocity experiment is assumed to satisfy the conditions listed below:

a. Initially the liquid mixture consists of two phases of the same solute and solvent with different coneentrations of the solute and separated by an indefinitely sharp interface (step-distribution).

b. The solute and the solvent are both mono-disperse nonelectrolytes and completely miscible.

c. The mixture has a negligible compressibility and shows no viscous effects or convection during ultracentrifugation.

d. The temporature is constant. The angular velocity is increased rapidly to the desired lovel and remains constant till the enr of the expcriment.

e. During the experiment the coneentration profile must not be affected by the presence of the tangential walls (bottom and meniscus).

f. A sector cell is used.

g. The refractive index depends linearly on the concentration of the solute. 
h. The transport coefficients of the solute are virtually independent. of the concentration in the range occurring in the system, thus $S$ and $I$ ) are independent of concentration.

i. The gratient of the refractive index is registered as a function of time and radial distance.

Having specified the experimental conditions, the equation of the concontration gradient of the solute as developed by Gehatia can be introduced:

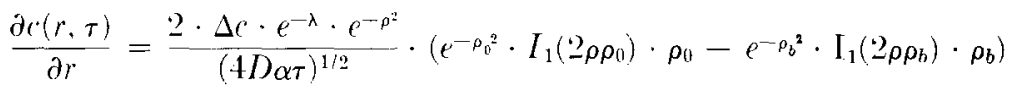

where:

$r \quad$ radius (the distance to the center of rotation)

$\tau \quad$ time elapsed since the beginning of the experiment $=t-t_{0} ; t$ is the time with an arbitrary origin; $t_{0}$ is the time at the start of the experiment

$r_{0} \quad$ initial position of the interface

$r_{b} \quad$ position of the bottom

c concentration of the solute

Ar initial concentration difference between both phases

$\lambda \quad=2 \beta \tau=2 \cdot \omega^{\prime} \cdot \omega^{2} \cdot \tau$,

$\omega$ is the angular velocity.

$$
\begin{array}{ll}
\alpha & =\left(e^{\lambda}-1\right) / \lambda, \\
\prime \prime & =r /(+D \alpha \tau)^{1 / 2}, \\
\rho_{\prime \prime} & =r_{1} e^{\lambda / 2} /(+D \alpha \tau)^{1 / 2}, \\
\rho_{\prime \prime} & =r_{b} e^{\lambda / 2} /(+D \alpha \tau)^{1 / 2}, \\
I_{1}\left(2 \rho \rho_{0}\right) & =\sum_{k=0}^{\infty}\left(\rho \rho_{0}\right)^{2 k+1 /}\left[k^{\prime \prime} !(k+1) !\right] .
\end{array}
$$

(Bessel function of the first kind and the first order.)

In the original rerivation of $E_{1}$. (1) the initial coucentration of the solute in one of the phases was equal to zero. In App. A it is proved that Ei. 11 is also valid in the more general case of an arbitrary concentration difference between the phases.

Equation (1) can be simplified considerably if the molecules of the solute are not too small and the duration of the experiment is relatively short. Under these circumstances the influence of the bottom on the concentration profile can be neglested [sec (11): 


$$
\rho_{0} \cdot e^{-\rho_{v}{ }^{2}} \cdot I_{1}\left(2 \rho \rho_{0}\right) \gg \rho_{b} \cdot e^{-\rho_{b}^{2}} \cdot I_{1}\left(2 \rho \rho_{b}\right) .
$$

In the second place, the argument $\left(2 \rho \rho_{0}\right)$ is normally large enough $\left(2 \rho \rho_{0}>\right.$ $\left.4 \times 10^{2}\right)$ to approximate $I_{1}\left(2 \rho \rho_{0}\right)$ by the first term of an appropriate asymptotic power expansion series [see (7), Sec. 9.7]:

$$
I_{1}\left(2 \rho \rho_{0}\right) \simeq\left[e^{2 \rho \rho_{0}} /\left(1 \pi \rho \rho_{0}\right)^{1 / 2}\right] \cdot\left(1-\frac{3}{16 \rho \rho_{0}}-\cdots\right)
$$

Substitution of the simplifications (2) and (3) into Eq. (1) gives:

$$
\frac{\partial c(r, \tau)}{\partial r}=\frac{\Delta c \cdot e^{-\lambda}}{(4 \pi D \alpha \tau)^{1 / 2}} e^{-\left(\rho_{0}-\rho^{2}\right.}\left(\frac{\rho_{0}}{\rho}\right)^{1 / 2} .
$$

The next step is to replace the concentration gradient by the refractive index gradient. The height $h(r, \tau)$ of a recorded sedimentation curve depends linearly on the concentration gradient:

$$
h(d, \tau)=h(r, \tau)=F \cdot \frac{\Delta n}{\Delta c} \cdot \frac{\partial c(r, \tau)}{\partial r},
$$

where

$$
r=G \cdot d+r_{l}
$$

$G$, radial magnification factor; $d$, radial distance of a point of a recorded curve with respect to a reference line; $r_{l}$, position of reference line; $\Delta n$, initial difference of refractive indices of the phases; $F$, vertical magnification factor. Eliminating the concentration gradient and replacing $\lambda, \rho$ and $\rho_{n}$ in Eq. (4) by the right sides of (1.1) to (1.3) gives the following equation:

$$
h(r, \tau)=\frac{F \cdot \Delta n}{(2 \pi)^{1 / 2}} \cdot \frac{r^{-2 \beta \tau}}{2 D \alpha \tau} \cdot \exp \left[-\frac{\left(r-r_{0} e^{\beta \tau}\right)^{2}}{4 D \alpha \tau}\right] \cdot\left(\frac{r_{0} e^{\beta \tau}}{r}\right)^{1 / 2} .
$$

Transformations. Equation (6) can be used as a model in the computation of $\beta$ and $D$ assuming the refractive index gradient is measured as a function of radial distance and time. The quantities $r_{v}, \Delta n$ and $F$ must also be determined. However, in doing so two difficulties arise. Firstly, Eq. (6) has not a suitable form to calculate $\beta$ and $I$ easily. Secondly, an aceurate experimental determination of $r_{0}$ and $\tau$ is hardly possible due to some unavoidable mixing near the interface as the start and to an uncertainty with respect to the exact moment of interface formation. These difficulties can be solved by means of two transformations. The first one dissolves the necessity of measuring $r_{1}, F$ and $\Delta n$. The second transformation allows the use of an arbitrary origin of the time axis 
by the introduction of a roference time $\tau_{r}$ to be sclected by the inrestigator.

The first transformation replaces $\beta, D, F, \Delta n, r_{0}$ and $\tau$ by a new set of time-dependent parameters $m_{0}, m_{1}$ and $m_{2}$ (shortly the vector $\mathbf{m}$ ):

$$
\begin{array}{ll}
m_{0}=F \cdot \Delta \| \cdot r_{0}{ }^{2} /(2 \pi)^{1 / 2} & m_{0}>0, \\
m_{1}=r_{0} \exp \left(\beta_{\tau}\right) & m_{1}>0, \\
m_{2}=2 D \alpha \tau & m_{2}>0 .
\end{array}
$$

The parameters $m_{1}$ and $m_{2}$ are related to the reduced moments of the concentration gradient with respect to the radius:

$$
\begin{aligned}
& m_{1}=\left(\bar{w}_{i}\right)^{1 / 2} \\
& m_{2}=\left(\tilde{w}_{4}-\bar{w}_{2}^{2}\right) / 2 \bar{w}_{2},
\end{aligned}
$$

where $\bar{w}_{i}$ is a reduced moment of order $i$ [see (1) for the definition]. Application of the first transformation to Eq. (6) yields:

$$
h(r ; \mathbf{m})=m_{0} \cdot m_{1}^{-2} \cdot \exp \left[-\left(r-m_{1}\right)^{2} / 2 m_{2}\right] \cdot\left[m_{1} /\left(m_{2} \cdot r\right)\right]^{1 / 2}
$$

The variables $h(r ; \mathbf{m})$ and $r$ are measurable quantities from which the parameter vector $\mathbf{m}$ can be derived.

The sccond transformation relates the components of the $\mathbf{m}$ vector to the transport cocficients via a new set of parameters. As the components of $\mathrm{m}$ are time dependent a subseript $i$ is attached to refer to discrete time $\tau i$. The subscript $r$ indicates the reference time and its corresponding parameter values, $m_{1, r}$ and $m_{2,2}$.

$$
\begin{aligned}
& Z_{(1, i}=\tau_{i}-\tau_{r} \\
& Z_{1, i}=2 \beta Z_{1(1, i}=\ln \left(m_{1, i} / m_{1, r}\right)^{2} \\
& Z_{2, i}=2 I D Z_{0, i}=\left(\frac{Z_{1, i}}{r_{1, i}-1}\right) \cdot\left(m_{2, i}-m_{2, r} \cdot \epsilon^{Z_{1, i}}\right) .
\end{aligned}
$$

The time derivatives of $Z_{1}$ and $Z_{2}$ are independent of the time and are equal to the transjort coefficients times a factor two.

The original problem to deduce $\beta$ and $D$ from the experimental data has been separated in two subproblems:

a. Determination of $\mathbf{m}_{i}$ from the experimental data.

b. Computation of $Z_{1}$ and $Z_{2}$ and their time derivatives, after selecting a reference time and a corresponding $\mathbf{m}$ rector. Both subproblems ran be solved by the methor of least scquares as described in Sect. III.

Onee the transport coefficients are known, the molecular weight of the solute follows immediately from the Svedberg equation (5): 


$$
M=\frac{R T S}{\left(1-\rho v_{1}\right) D}\left(\frac{\partial \ln \alpha 1}{\partial \ln c 1}\right)_{T}
$$

where

$S \quad \beta / \omega^{2}$

$R \quad$ gas constant

$T$ absolute temperature

$\rho \quad$ average density of the solution

$v_{1} \quad$ partial volume of the solute

$\alpha_{1} \quad$ activity of the solute $\}$ both expressed in gmoles $/ \mathrm{cm}^{3}$

$c_{1}$ concentration of the solute $\}$ or $\mathrm{g} / \mathrm{cm}^{3}$

The Eqs. (7), (8), (9) and (10) form the model to be applied to the data processing.

\section{METHOD OF IFAST SQUARES}

\section{Derivation of the $\mathbf{m}$ vector}

During an experiment a certain number of refractive index gradient curves are registered. Each curve must be represented numerically, which can be done by measuring the moment of recording $t$, the radial magnification factor $G$ and the coordinates of at least three points on a curve: the height $y$ with respect to the base line and the radial distance $d$ relative to a reference line. The product of $G$ and $d$ added to the distance $r_{l}$ between the reference line and the center of rotation, is the actual distance $r$ of a point on a curve to the axis of rotation. It is convenient to select the points radially equidistant. The zero time may be chosen arbitrarily. Then the outcome of an experiment is given by the following set of data:

$$
\begin{aligned}
\left(y_{k}, r_{k}\right)_{i}, t_{i} \quad k & =1, \ldots, K_{i} \quad\left(K_{i} \geqslant 3\right) \\
i & =i, \ldots, I .
\end{aligned}
$$

The subscript $k$ refers to a selected point; the subscript $i$ refers to a curve. These data suffice to determine a $\mathrm{m}$ vector for each curve. As the derivation is identical for each curve, the suffix $i$ will be deleted from now on.

The connection between the experimental data and the theoretical model is made by relating the experimental height $y_{i}$ to the predicted height $h\left(r_{k} ; \mathrm{m}\right)$ is given by Eq. (8):

$$
y_{k}=h\left(r_{k} ; \mathbf{m}\right)+\epsilon_{k} \quad k=1 . \ldots, K .
$$

The residue $\epsilon_{k}$ accounts for the discrepancy between the model and reality. In order to find the closest fit of the model to the experimental 
Aata the sum of square residues is minimised by adjusting the vector $\mathbf{m}$. The sum of square residuce labbr. SSRI reads:

$$
Q(\mathbf{m})=\sum_{k=1}^{K} \epsilon_{k}{ }^{2}=\sum_{k=1}^{K}\left(y / k-h\left(r_{k} ; \mathbf{m}\right)\right)^{2}
$$

$Q$ is a positive continuous, twice differcutiahle scalar function of $\mathbf{m}$, defined on $V$, i.e., $m_{n}>0, m_{1}>0, m_{2}>0$. A local minimum exists at $\mathbf{m}^{\prime \prime}$ ${ }_{\epsilon} V$ when the following conditions are satisfied at $\mathbf{m}=\mathbf{m}^{n}$ :

(1) $\frac{\partial Q(\mathbf{m})}{\partial m}=0 \quad j=0,1, \stackrel{2}{-}$.

(2) The matrix of second order derivatives of $Q$ with respect to $\mathbf{m}$ is positive definite.

An analytical approach to the solution of $(13.1)$ is not very promising due to the nonlinear occurrence of the parameters $m_{1}$ and $m_{z}$ in the expression of $\left.h_{1} r_{k} ; \mathrm{m}\right)$. Application of 13.11 yideds symunterical expressions after some substitutions:

$$
\sum_{k=1}^{K} y_{k} \cdot h\left(r_{k} ; \mathbf{m}\right) \cdot\left(r_{k}\right)^{j}=\sum_{k=1}^{K}\left(h\left(r_{k} ; \mathbf{m}\right)\right)^{2} \cdot\left(r_{k}\right)^{\prime} \quad i=0,1,2 .
$$

During the operations the quantity $h\left(r_{k} ; \mathbf{m}\right)$ has been temporarily replaced by the right side of $\mathrm{E}$ (q. 181 . This set of equations is not casily solvable either. This problem howerer, minimising a function of three variables, can be handled numerically by assmming the SSR to be an unimodal function on $T$ and the matrix of second order derivatives is positive definite at the minimum." Numerical optimalisation techniques [e.g., direct climbing routines, see 161 chap. $7 \mid$ deal with this class of problems successfully.

A simple but effeetive routine called SEARCII has been developed. The SSR is minimised by means of a sorics of cxplorations in a subset of $V$. The search to a minimum can be mate more efficient by reducing the dimension of the siR. The rector component $m_{1}$ being a linear parameter in Eq. 18 ) can be eliminated from $\mathrm{E}_{\mathrm{q}} .(12)$ by means of condition (13.1i, ase $j=0$. This yiclds:

"We are not able to prove llense assumptions. No experimental evidene has bern found to distrust the validity of the unimodality of the SSR. Nevertholess this point should twe kerpt in mind whon inspocting the numerical msults. A theretial analysis of the properties of the matrix of the second order devivatives does not produce very muth as the ealeulations become very complicated. 


$$
Q\left(m_{1}, m_{2}\right)=\sum_{k=1}^{K}\left(y_{k}-f\left(r_{k} ; m_{1}, m_{2}\right) \cdot m_{0}^{*}\right)^{2}
$$

where

$$
f\left(r_{k} ; m_{1}, m_{2}\right)=h\left(r_{k} ; \mathbf{m}\right) / m_{0}
$$

and

$$
m_{0}{ }^{*}=\sum_{p=1}^{K} y_{p} \cdot f\left(r_{p} ; m_{1}, m_{2}\right) / \sum_{p=1}^{K}\left(f\left(r_{p} ; m_{1}, m\right)_{2}\right)^{2}
$$

The variable $Q\left(m_{1}, m_{2}\right)$ is a positive, continuous, twice differentiable scalar function of $m_{1}$ and $m_{2}$, defincd for $m_{1}>0$ and $m_{2}>0$. The variable $m_{0} *$ is also a function of $m_{1}$ and $m:$. Equation (14.1) is the objective function of the SEARCH routine. Appendix $B$ describes the various activities of this module.

At the end of the optimalisation the routine produces the minimised sum of square residues ( $Q$ min) and the optimal values of $m_{1}$ and $m_{2}$. The corresponding value of $m_{0}^{*}$ is calculated via (14.2). Finally a new parameter $m_{: 3}$ is introduced representing the average seatter of the experimental points along the optimal curve:

$$
m_{3}=\left[Q \mathrm{~min} / K \cdot\left(m_{0}^{*}\right)^{2}\right]^{1 / 2} .
$$

The division by the square of $m_{0}{ }^{*}$ is necessary to eliminate the influence of the vertical scaling factor.

The procedure as described in this part of the section has to be repeated for each curve. The obtained set of $\mathbf{m}$ vectors, $\mathbf{m}_{i}, i=1, \ldots$, $I$ will be used to derive the transport coefficients.

\section{Computation of $S$ and $D$}

The second transformation $(9)$ establishes the relation betwcen the transport coefficients and components of the $\mathbf{m}_{i}$ vectors. Firstly, the $Z$ values are calculated. This calculation requires the reference parameters $m_{1, r}$ and $m_{:, r}$. The parameters of the curve having the smallest scatter (i.e., the smallest $m_{;}$value of all curves) are taken as references. The factors $Z_{1, i}$ and $Z_{2, i}(i=1, \ldots ., I)$ follow straight forward from Eq. (9). Then the tince derivatives of the stries $Z_{1, i}$ (equal lo $2 S_{\omega}{ }^{2}$ ) and $Z:, i$ (equal to $2 D$ ) can be calculated by the method of weighted least squares. Only one calculation will be given because the derivations are identical in both cases.

Owing to the second transformation a linear relationship between a $Z$ factor and the experimental time $t$ is introduced: 


$$
Z_{i}=A+B t_{i}+\mu_{i} \quad i=1, \ldots, I,
$$

where

$Z_{i} \quad$ represents either $Z_{1, i}$ or $Z_{2, i}$

li $\quad=2 S \omega^{2}=2 \beta$ in case of sedimentation

$B \quad=2 D$ in case of diffusion

A is a quantity depending on the origin of the time axis

$\mu_{i} \quad$ is a deviation due to the approximate nature of the model and to posisible errors in the computed $Z$ values

The sum of the weighted squiare residues is given by:

$$
R(A, B)=\sum_{i=1}^{I} w_{i} \cdot \mu_{i}^{2}=\sum_{i=1}^{I} w_{i} \cdot\left(Z_{i}-A-B \cdot t_{i}\right)^{2} .
$$

The symbol $u_{i}$ denotes a weight factor. The weight factors will be used to suppress the contribution of those observations showing relatively much seatter. Therefore a (rather arbitrary) inverse relation between the weight fartors and the scatter factors is applied:

$$
w_{i}=\left(1-m_{3, i} / \sum_{i=1}^{l} m_{3, i}\right) /(I-1) \quad I \geqslant 2 .
$$

The parameters $A$ and $B$ can be derived by minimising $R(A, B)$. Application of the necessary conditions for the existence of a minimum $\left(A^{\circ}, B^{\circ}\right)$ yields:

$$
\begin{aligned}
& A^{0}=\bar{Z}-B^{0} \cdot \bar{t} \\
& B^{0}=(\overline{Z t}-\bar{Z} \cdot \bar{t}) / N
\end{aligned}
$$

where

$$
\begin{aligned}
N & =\bar{t}^{2}-(\bar{t})^{2}, \\
\overline{Z t} & =\sum_{i=1}^{I} w_{i} Z_{i} t_{i}, \\
\overline{t^{2}} & =\sum_{i=1}^{\zeta} w_{i} t_{i}{ }^{2}, \\
\bar{t} & =\sum_{i=1}^{I} w_{i} l_{i}, \\
\bar{Z} & =\sum_{i=1}^{I} w_{i} Z_{i} .
\end{aligned}
$$


It is easy to prove that the matrix of second order derivatives is definite positive in $\left(A^{\circ}, B^{\circ}\right)$. Furthermore, it should be noted that $N, B^{0}$ and $R\left(A^{\prime \prime}, B^{\prime \prime}\right)$ are invariant to uniform translations of the time axis.

The sample error of $B^{\prime \prime}$ is given by the formulae [see (8)]:

$$
\begin{aligned}
\sigma\left(B^{0}\right) & =\left[R\left(A^{0}, B^{0}\right) / N(I-2)\right]^{1 / 2} & & I>2 \\
& =0 & & I=0 .
\end{aligned}
$$

As soon as both transport coeffieients are known the molecular weight $M$ can be calculated via $\mathrm{E}_{1}$. (10) .

\section{APPLICATIONS AND DISCUSSION}

In order to test the performance of the numerical solution method threc sets of artificial "experimental data" have been generated and procesced." These data can be considercd as being sampled from artificially constructed curves. The first test dealt with curres satisfying the model [the E(s. (8) and (10) ] exactly. In the second test the eurves of the first case were shifted upward with respect to the base line (about 5\% of the maximum heighti. This test accounts for those situations in which the height of a curve has a svstematic deviation due to an uncertainty in the position of either the base line or the curve. In the third test the right sides of the curves have been omitted in order to check the sensitivity of the method to incomplete data. Table 1 lists the input values of the $\mathbf{m}$ vectors from which the curves have been constructed and the output values of the $\mathbf{m}$ rectors as derived by the SEARCH routine. Component $m_{1}$ is well reproduced and not very sensitive to inaccurate data. Component $m$. is also well reproduced in the first test case; in the other tests moderate systematic deviations occur. In the sccond test the SEARCH tries to minimise the deviation from the model by making $m_{2}$ systematically greater than the original input value. The absence of the right sides of the curves apparently causes a systematic underestimation of $m_{22}$. The output values of $m_{0}$, calculated according to (14.2), show a rather strong correlation with $m_{2}$. The consistent offset produced in the first curve of each ease is caused by the very sharply peaked curves (ratio of lowest to highest value is $1.0 \mathrm{E}-14$ ) and to the small number of specified coordinates (five).

The transport coefficients and their deviates are calculated from the

${ }^{3}$ A digital program has been developed named DISEMO. The size of the compiled program is about $9 \mathrm{~K}$; the execution time for the processing of the data of one experiment varies between 5 and 15 sec. Requests for a listing of the source module and a sample input form should be addressed to: Polymer Chemistry Laboratory, Department of Chemiral Enginering. Twente University of Technology, Enschede, The Netherlands. 


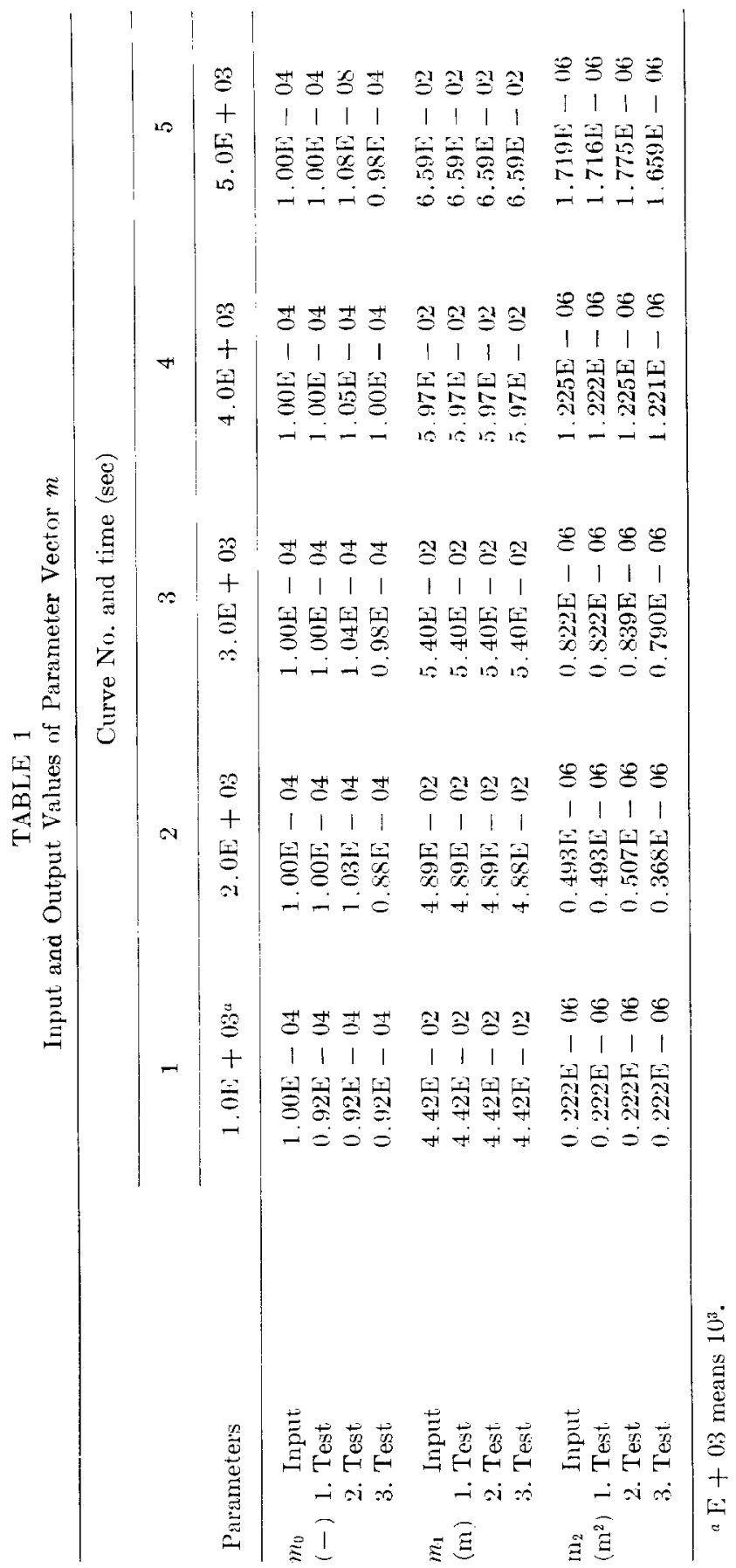


TABLE 2

Input and Output Values of Physical Variables ${ }^{a}$

\begin{tabular}{llcccc}
\hline Physical quantity & Input & 1. Test & 2. Test & 3. Test \\
\hline$S$ & $\left(\mathrm{sec}^{-1}\right)$ & $2.46 \mathrm{E}-11$ & $2.46 \mathrm{E}-11$ & $2.46 \mathrm{E}-11$ & $2.46 \mathrm{E}-11$ \\
$\sigma(S)$ & $\left(\mathrm{sec}^{-1}\right)$ & - & $4.79 \mathrm{E}-15$ & $6.64 \mathrm{E}-15$ & $6.38 \mathrm{E}-15$ \\
$D$ & $\left(\mathrm{~m}^{2} / \mathrm{sec}\right)$ & $1.00 \mathrm{E}-10$ & $1.00 \mathrm{E}-10$ & $1.03 \mathrm{E}-10$ & $0.98 \mathrm{E} 10$ \\
$\sigma(D)$ & $\left(\mathrm{m}^{2} / \mathrm{sec}\right)$ & - & $0.18 \mathrm{E}-12$ & $2.16 \mathrm{E}-12$ & $5.43 \mathrm{E}-12$ \\
$M$ & $(\mathrm{~kg} / \mathrm{kmole})$ & $2.195 \mathrm{E}+05$ & $2.200 \mathrm{E}+05$ & $2.13 \mathrm{E}+05$ & $2.23 \mathrm{E}+05$ \\
\hline
\end{tabular}

a Numerical values of the various constants used in the test computations: $r_{0}=$ $4.0 \mathrm{~F}-02(\mathrm{~m}) ; \omega=6.09 \mathrm{E}+04(\mathrm{rpm}) ; \rho=1.0\left(\mathrm{~g} / \mathrm{cm}^{3}\right) ; \bar{v}=0.75\left(\mathrm{~cm}^{3} / \mathrm{g}\right) ; T=298.15$ $\left({ }^{\circ} \mathrm{K}\right) ;(\partial \ln \alpha / \partial \ln c)_{T}=0.9$.

output values of $m_{1}$ and $m_{2}$ as described in the second part of Sect. III. Table 2 lists the preassigned values and the reproduced values of the transport coefficients and the molecular weight. The valucs of the auxiliary physical quantities used in the computation of $M$ are given in footnote $a$ of Table 2. The sedimentation coefficient is not very sensitive to disturbances. Even in the worst case (third test) the relative crror remains smaller than $0.1 \%$. The diffusion coefficicnt and its sample error are much more affected by the upsets. The systematic reviations of $m_{2}$ in the second and third test ease (see Table 1 ) propagate into the diffusion coefficient, but the second transformation and the weighted least squares method smooth the seatter to some extent.

These tests with artificial "experimental" data give an indication of the accuracy of the mothod under various circumstances. But the simultaneous effect of all sources of upsets, i.c., numerically, experimentally and inadcquacies of the model can only be observed from real experimental data. Table 3 lists the experimental conditions and the calculated $\mathbf{m}$ vectors including the normalized weight factors of a test with $\alpha$-chymotrypsinogen. Table 4 is a survey of the calculated transport coefficients, their sample errors and the molecular weight together with some corresponding data from the literature. The answers obtained by the method of least squares are in satisfactory agreement with the data found in the literature. The numerical errors do not prevail over the experimental inaccuracies. There are some indications that the overall accuracy has been improved by applying this method.

\section{DISCUSAION}

The diseussion will be restricted to the presented method for data processing and the applicability of the model. The experimental procedure itself is not considered in the discussion because this procedure is common to each data processing method. 


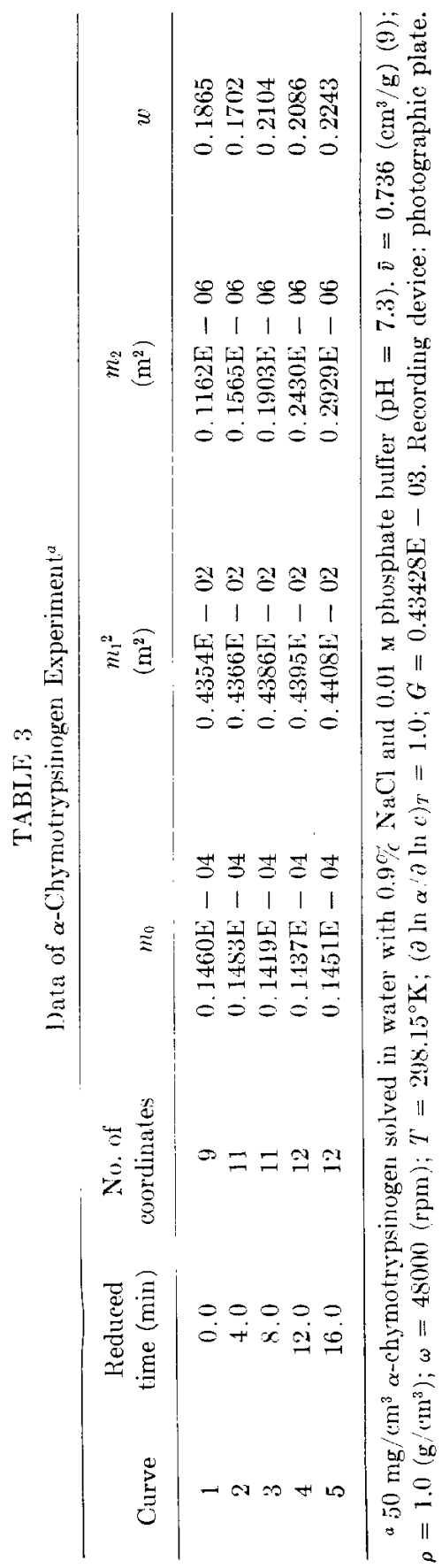




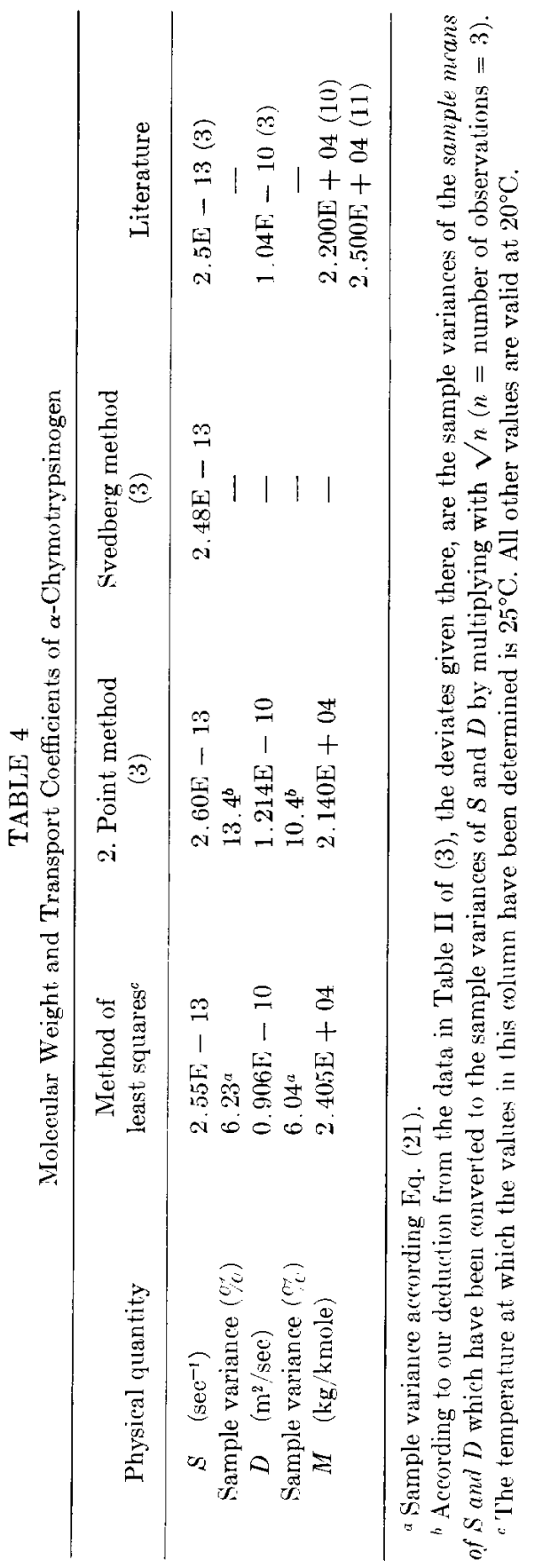


Problems with the proposed method may arise at three stages:

a. In sampling data from the recorded curves.

b. When applying the mathematical model ontside its ralidity ranger.

(. During the mumerical operations.

The main difficulties in simpling data concern mainly the accuracy of the ordinates of the selecterl points along a curve. The precision ean become rather low, especially near the tails of a curve. It is recommended to sample only at the top and the sides of a curve. The difference in accuraty of the virious points could be compensated by asigning wright factors. As no reliable method was known to convert the relative accuracy into a weight factor, in our method all points have the same weight.

The most important violations of the assumptions underlying the mathematical model are threefold. The purity and the mono-dispersity of the solute is sometimes cluestionable. In that case the basic equations of the model are strictly taken no longer appliablo and maintaining the morlel as the base of the method rields less reliable results. An experimental investigation of these effects may rarify the quantitive asperes of this problem. The deviations from the assumed initial concentration distribution and the oecurrenes of boundary effects are the second and third source of errors. With increasing run time the consequences of the initial disturbanecs derease while the boundary effects become more important. A compromise in run length must be found. This sort of model deviations however are inherent to arch model hased on Gehatia: mothod.

The rrucial points in the numerical operations are:

a. The effectiveness of the optimalisation.

b. The use of reference parameters in the second transformation.

(. The mather arbitrary definition of the curve weight factors.

d. The assumption that $S$ and $D$ are constant over the concentration lange omployed.

The cfectiveness of the optimalisation is an important factor as the optimalisation results are the starting point for the following numerical operations. The applied routine SEARCH works sufficiontly accurately and ritrilly. but it is not essential for the methor. It can be replaced by any other loutine according to the preference of the user.

The application of the reference parameters introduces the potential danger of loss of numerical areuracy because two numbers of the same order of mugnitude are subtracted in the second transformation. Sucondly. systematio deviations may oceur due to incorrect ralues of the reference 
parameters. This only applies to the $Z_{i, i}$ factors as can be seen from (9.2) and (9.3):

$$
\begin{aligned}
& \frac{d Z_{1, i}}{d t} \text { is independent of } Z_{1, r} \text {, } \\
& \frac{d Z_{2, i}}{d t} \text { has a dependency on } Z_{1, r} \text { and } Z_{2, r} \text {. }
\end{aligned}
$$

The investigated problems gave no indications that these points had a serious bearing on the results, but they should be mentioned for sake of completeness.

As stated above the definition of the curve weight factors is an arbitrary choice, but it provides a means to account for the relative accuracy of each curve. A different definition would produce different numerical values for the weight factors. However, the selection of the reference curve is hardly affected by the definition. The influence of the value of the weight factors on the transport coefficients has been investigated for the $\alpha$-chymotrypsinogen experiment. A repetition of the calculations with equal weights and the same reference curre vields: $S=2.59 S$ and $D=0.903 \times 10^{-11}\left(\mathrm{~m}^{2} / \mathrm{sec}\right)$. The differences with the values in Table 4 are rather small and ran almost be neglected with respect to the sample errors.

The applicability of the method of least squares is not restricted to the model as presented in Sect. II. Under certain conditions (very small sedimentation coefficient) the factor $\alpha=\left(e^{2 \beta i}-1\right) / 2 \beta t$ becomes virtually equal to one and can be left out. In this ease the definition of $Z_{2 . i}(9.3)$ simplifies to $Z_{2, i}=m_{2, i}-m_{2, r}$. It is also possible to retain more terms of the power series expansion [see Eq. (3) |. The transformations do not change.

It should be noted that the method allows us to compare predicted values of $r_{0}, t_{n}$ and $F \cdot \Delta n$ with observed values. After the derivation of $s$ and $D$ the quantities $r_{0}$ and $t_{0}$ can be calculated from the definitions of $m_{1}$ and $m_{2}$. Applying the definition of $m_{0}$ yields the product of $F \cdot \Delta n$. This provides a means to check for a possible discrepancy between model and reality at the initial stage of an experiment.

\section{CONCLUSIONS}

In the description of the least squares method the mathematical aspects have been more emphasised than the experimental sides. Summarising the features, the method requires neither the difficult to observe quantities $r_{0}$ and $t_{0}$ at the top or the area of a refractive index gradient curve. but uses sampled curve heights around the top. Apart from the transport constants and the molecular weight it produces also the same errors of the constants. The performance of the method with respect to the re- 
producibility is good. As far as the method has been tested on experimental sedimentation data, the results are encouraging. Therefore, it may be an useful tool for the processing of sedimentation velocity data of proteins. where $S$ and $D$ are independent of the concentration.

\section{APPLNDIX A: EQUATIONS OF THE CONCENTRATION GRADIENT}

In this appendix it is shown that $\mathrm{E}_{1}$. (1) of soct. If is valid for a two-component system, initially consisting of two solutions with a conrentration difference. This includes also a two-component system initially with a pure solvent phase and a solution.

The sedimentation process in an infinite sector cell is described by the following set of equations:

$$
\left.\frac{\partial c(r, \tau)}{\partial \tau}=\frac{1}{r} \frac{\partial}{\partial r}[r \cdot I) \cdot \frac{\partial r(r, \tau)}{\partial r^{r}}-\beta \cdot r^{2} \cdot c(r, \tau)\right] .
$$

Initial conditions:

$$
\begin{array}{lll}
c(r, 0)=c_{0}>0 & \tau=0 & 0<r \leqslant r_{0} \\
c(r, \theta)=c_{1} \geqslant c_{11} & \tau=0 & r_{0}<r
\end{array}
$$

Bomdary conditions:

$r(r, \tau)$ and $\frac{\partial c(r, \tau)}{\partial r}$ both finite and continuous for all

$$
r>0 \text { and all } \tau>0 .
$$

The concentration and the concentration gradient can be calculated from this bountary ralue problem.

By means of a transformation of variables this boundary ralue problem is converter into a boundary problem that has already been solved |Fujita (4). Chap. 2.5|. The transformation is very similar to the one ilphlied by Fujita when solving the problem (A.1) in the (ase $c_{1}=0$.

$$
\begin{aligned}
\lambda & =2 \beta \tau, \\
\epsilon & =2 I) / \beta r_{0}{ }^{2}=1 /\left[\rho_{01}{ }^{2} \cdot\left(1-e^{-\lambda}\right)\right], \\
\chi & =\left(r / r_{0}\right)^{2}=\left(\rho / \rho_{1}\right)^{2} e^{\lambda \lambda}, \\
\theta(\chi, \lambda) & =\frac{c(r, \tau)-c_{0} \cdot e^{-\lambda} .}{c_{1}-c_{0}} .
\end{aligned}
$$

The cualities $\rho$ and $\rho_{n}$ have been defined in Sect. II.

The new boundary value problem reads:

Initial condition:

$$
\frac{\partial \theta(\chi, \lambda)}{\partial \lambda}=\frac{\partial}{\partial \chi}\left[\chi\left(\epsilon \frac{\partial \theta(\chi, \lambda)}{\partial \chi}-\theta(\chi, \lambda)\right)\right] .
$$

$$
\begin{array}{lll}
\theta(\chi, 0)=0 & \lambda=0 & \chi<1, \\
\theta(\chi, 0)=1 & \lambda=0 & \chi>1 .
\end{array}
$$

Boumlary ronditions: similar to those of (A.1). 
Fujita (4) gives the solution of (A.3). Returning to the original variables yiclds an expression for $c(\rho, \lambda)$ :

$c(\rho, \lambda)=c_{0} e^{e^{-\lambda}}+\left(c_{1}-c_{0}\right) \cdot \epsilon^{-\lambda} \cdot\left[1-2 \cdot \int_{0}^{\rho_{0}} \gamma \cdot e^{\rho^{2}} \gamma^{\circ} \cdot I_{0}(2 \gamma \rho) d \gamma\right]$.

By putting $c_{n}$ equal to zero this equation becomes identical to the solution given by Fujita (4), Gchatia and Katchalski (12) in the case $c_{0}=0$.

Evaluation of the right side of (A.4) gives:

$$
c(\rho, \lambda)=c_{0} e^{-\lambda}+\left(c_{1}-c_{0}\right) e^{-\lambda} \cdot\left[\int_{\rho_{0}}^{\infty} 2 \cdot \gamma \cdot e^{-\rho^{2}-\gamma^{2}} \cdot I_{0}(2 \gamma \rho) d \gamma\right] .
$$

Assuming the presence of the bottom does not yet affect the sedimentation process, the upper boundary of the integral can be replaced by $p$ (defined in Sect. II) :

$$
c(\rho, \lambda)=c_{0} e^{-\lambda}+\left(c_{1}-c_{0}\right) e^{-\lambda} \cdot\left[\int_{\rho n}^{\rho b} 2 \cdot \gamma \cdot e^{-\rho^{2}-\gamma^{2}} \cdot I_{0}(2 \gamma \rho) d \gamma\right] .
$$

The derivative of this equation with respect to $r$ gives Eq. (1) of sect. II. Gehatia (1) proves this assertion in the ase $c_{n}=0$. As the calculations are almost identical for $c_{n}>0$ the derivation is not repeated here.

\section{APPENDIX B: SEARCH ROUTINE}

The SEARCH routine minimises the objective function values by varying the independent variables, $m_{1}$ and $m_{\text {... }}$. The module has three main parts: (a) initiation; (b) explorations; (c) termination.

The first scction establishes the starting point for the first exploration $\left(m_{1}{ }^{s}, m_{2}{ }^{*}\right)$ and the initial step sizes $\left(\Delta m_{1}, \Delta m_{2}\right)$. The quantities $m_{1}{ }^{*}$ and $m_{2}{ }^{*}$ are derived from Eq. (8) for which purpose the left side has been put equal to the experimental height $y_{k}$. After some rearrangcments the following equations are obtained:

$$
\begin{aligned}
& m_{2^{8}{ }^{8}}=\Delta r^{2} /\left[\ln \left(y_{k}{ }^{2} /\left(y_{k 1} \cdot y_{k+1}\right)\right)+0.5 \ln \left(r_{k}{ }^{2} /\left(r_{k+1} \cdot r_{k-1}\right)\right)\right], \\
& m_{1}{ }^{8} . k=r_{k}+m_{2^{8}{ }^{8}} \cdot\left[\ln \left(y_{k+1} / y_{k-1}\right)+0.5 \ln \left(r_{k+i} / r_{k-i}\right)\right] /(2 \cdot \Delta r),
\end{aligned}
$$

where

$$
\Delta r=r_{k+1}-r_{k}=r_{k}-r_{k-1} \quad(k<K \text { and } k \text { even }) .
$$

Any three equidistant points suffice to calculate $m_{1}{ }^{*}{ }_{k}$ and $m_{2}{ }^{*}{ }_{k}$. But the initial guess becomes more accurate when this computation is repeated for several sots of three points and the linear average is taken and applied to form the first base point $\left(m_{1}{ }^{*}, m_{2}{ }^{*}\right)$. The initial step size of $m_{1}$ has been set equal to $\Delta r$, while $\Delta m_{2}=0.1 m_{z}{ }^{*}$.

The second part controls the explorations. An exploration is defined by the actions listed below: 
a. Computation of the value of the objective function in a base point.

b. Computation of the coordinates and the value of the objective function in four points surrounding the base point. If any of these points coincides with a previously explored point then this point is skipped.

a. The point with the lowest value of the objective function becomes the next base point.

d. Eraluation:

Case 1: The new selected base point coincides with the previous onc. The step sizes are redueed proportional to the steepest slope found in the last cxploration. However, when the number of explorations will exced 50 or when all step sizes are already smaller than $0.1 \%$ of the actual magnitude of the corresponling independent rariables, then the control is trinsferred to the termination sertion. Otherwise at new exploration starts.

('ase 2: The new selested base point does not coincide with the previous one. The scareh will be continued unless the number of explorations exreeds 50 ipreventing excessive calculations). When two consecutive shifts of the base point in the same direction occur, the step size in that direction is doubled.

Figure 1 gives a graphical representation of a few explorations. The numbers assigned to the points indicate in which order the space

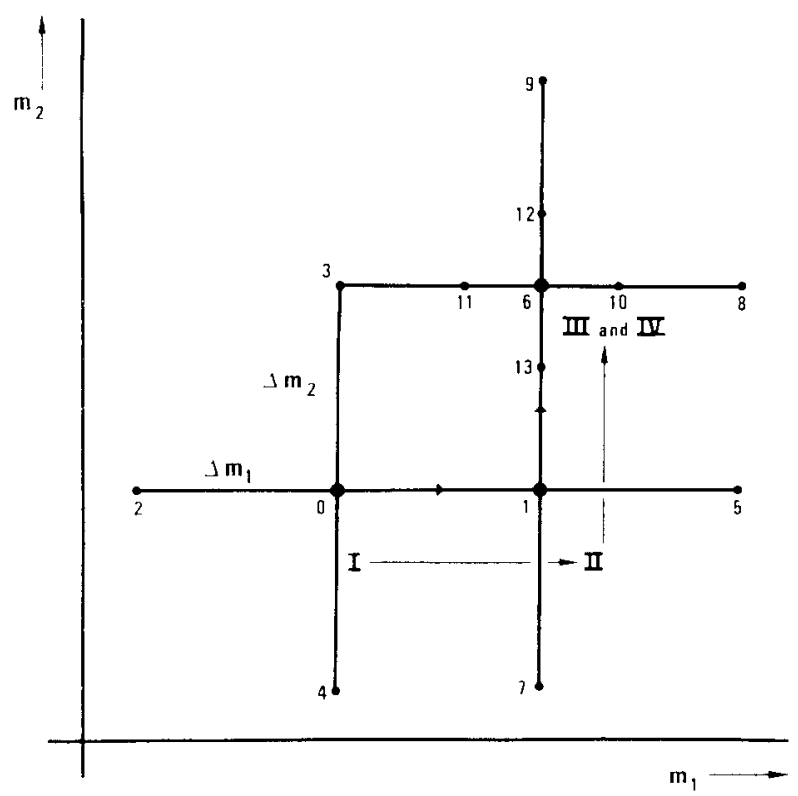

FIG. 1. Example of consecutive explorations. 
is explored. The sum of square residues is calculated in base point 0 . Afterwards the points $1,2,3$ and 4 follow. Suppose point 1 has the smallest sum, then point 1 becomes the second base point (II). Point 6 is both third and fourth base point, assuming the step sizes have been reduced between the third and fourth exploration.

The termination section declares the last explored base point to be the minimum. A message is given when the number of explorations exceeds 50 .

\section{ACKNOWLEDGMENT}

The authors thank Professor Schuyer (Dept. of Chemistry) for helpful discussions.

\section{REFERENCES}

1. Gehatia, M. (1959) Kolloid Z. U. Z. Polymere 167, 1.

2. Gehatia, M., and Hubner, L. (1960) Z. Naturforsch. B 15, 351.

3. Jaenicke, R., and Gehatia, M. (1960) Biochim. Biophys. Acta 45, 217.

4. Ftritsa, H. (1962) Mathematical Theory of Sedimentation Analysis, Academic Press, New York.

5. de Groor, S. R., aNd MazUr, P. (1963) Non-equilibrium Thermodynamics, NorthHolland, Amsterdam.

6. Wilde, D. J., ANo Berghrek, C. S. (1967) Foundations of Optimization, Prentice Hall, New York.

7. Rectorys, E. K. (1970) Survey of Applicable Mathematics, M.I.T. Press, Cambridge, MA.

8. Draper, N. R., and smith, H. (1966) Applied Regression Analysis, Wiley, N'x York.

9. Brandrup, J., and Immergut, E. H., (eds.) (1966) Polymer Handbook, Wiley (Interscience), New York.

10. H. M. Rauen (ed.), (1964), Biochemisches Tuschenbuch, 2nd ed., SpringerVerlag, Berlin.

11. Mann Rescarch Laboratory, New York.

12. Gehatia, M., And Katchalski, E. (1959) J. Chem. Phys. 30, 1334. 